\title{
PRODUTIVIDADE DE FELLER-BUNCHER EM POVOAMENTO DE EUCALIPTO EM RELEVO ACIDENTADO
}

\author{
Ezer Dias de Oliveira Júnior ${ }^{1}$, Fernando Seixas ${ }^{2}$, João Luis Ferreira Batista ${ }^{2}$ \\ ${ }^{1}$ Eng. Florestal, Dr., Fait/ACITA, Itapeva, SP, Brasil - ezer@ fait.edu.br \\ ${ }^{2}$ Eng. Florestal, Dr., Depto. de Ciências Florestais, ESALQ/USP, Piracicaba, SP, Brasil - fseixas@esalq.usp.br - parsival@usp.br
}

Recebido para publicação: 12/09/2007 - Aceito para publicação: 12/08/2009

\begin{abstract}
Resumo
Este estudo avaliou a produtividade de dois modelos de feller-buncher de esteiras, Timberjack 608L e Timbco 520, na atividade de corte de árvores de eucalipto em terreno acidentado, em um sistema de colheita de árvores inteiras. Através de um estudo de tempo, foi quantificada a influência da inclinação do terreno na produtividade das máquinas, em dois povoamentos florestais com diferentes produções. Foi proposto um modelo de predição para a produtividade do feller-buncher em função do padrão do povoamento e da inclinação do terreno, com o relevo influenciando cerca de $80 \%$ do seu desempenho efetivo.

Palavras-chave: Desempenho efetivo; produtividade da máquina; colheita de eucalipto.
\end{abstract}

\section{Abstract}

Feller-buncher productivity in eucalyptu's plantation on steep ground terrain. This study evaluated two feller-bunchers productivity, one Timberjack 608L and one Timbco 520, working on an eucalyptus's plantation located on a steep ground terrain. A time study quantified the slope influence, on two different sites in terms of forestry productivity, over machine production. The ground slope influenced up to $80 \%$ of feller-buncher productivity.

Keywords: Operacional performance; full-tree logging system; feller productivity.

\section{INTRODUÇÃO}

A produtividade de uma máquina de colheita de madeira depende de diversos fatores, dos quais se destacam: a) localização geográfica e extensão da área de trabalho; b) aspectos climáticos; c) capacidade de suporte do terreno; d) relevo; e) características das árvores; f) características da floresta e da colheita; e g) capacitação do operador (MALINOVSKI et al., 2002).

Um dos sistemas de colheita de madeira é chamado de árvores inteiras, envolvendo o corte das árvores e o seu transporte até outro local, geralmente a margem dos carreadores, para posterior processamento. Esse sistema pode ser composto por um feller-buncher, que corta e acumula árvores, depositando-as em feixes, e um skidder, que faz o arraste das árvores, podendo ser realizado tanto em local plano como acidentado (MALINOVSKI et al., 2002; SANT'ANNA, 2002).

As restrições ao uso de feller-bunchers dizem respeito à declividade do terreno, existindo um limite ao redor de $27^{\circ}$ de inclinação, com redução na capacidade efetiva de operação. As condições ótimas para o emprego de equipamentos de maior porte estão em declividades até $8^{\circ}$, um mínimo de 750 árvores por hectare e volume individual por árvore entre 0,23 e $0,28 \mathrm{~m}^{3}$. Nas declividades até $8^{\circ}$, a máquina trabalha melhor acompanhando os contornos do terreno. Em declives maiores, a máquina deve trabalhar morro acima ou abaixo (CONWAY, 1976).

Gingras (1988) avaliou os efeitos de alguns fatores de sítio e talhão no desempenho de um fellerbuncher de esteiras Timberjack Timbco 2518, com nivelamento de cabine $20^{\circ}$ lateralmente e $27^{\circ}$ frontal (apto a inclinações até $30^{\circ}$ ) e capacidade de diâmetro máximo de $51 \mathrm{~cm}$. As principais variáveis determinadas pelo autor foram número de árvores acumuladas por ciclo de corte e DAP médio, sendo que o declive afetou a produção quando excedeu $20^{\circ}(35 \%)$.

Para Valverde et al. (1996), o volume de madeira por hectare foi a principal variável de influência na operação de corte de árvores com feller-buncher, apresentando também um melhor 
desempenho em talhões com espaçamento um pouco maior, $3,0 \times 2,0 \mathrm{~m}$ contra $3,0 \times 1,5 \mathrm{~m}$. Visser; Stampfer (2003), avaliando a produtividade de um feller-buncher em segundo desbaste em uma plantação de pinus, concluíram que as principais variáveis de influência foram o volume por árvore e o número de árvores por feixe.

A determinação e quantificação dessas variáveis de influência são fatores determinantes para o planejamento florestal e o sucesso das operações, contribuindo sobremaneira com a otimização e a viabilidade econômica das atividades de colheita. $\mathrm{O}$ alto custo das máquinas florestais exige o seu emprego durante o maior número de horas possíveis e elevada produtividade, principalmente quando comparado com opções de menor aporte tecnológico, como o uso de mão de obra intensiva.

Este estudo avaliou a produtividade do feller-buncher atuando em um sistema de colheita de árvores inteiras, na atividade de corte de árvores de eucalipto em relevo acidentado, quantificando a influência da inclinação do terreno em condições de diferentes produtividades da floresta.

\section{MATERIAIS E MÉTODO}

\section{Materiais}

Local

Foi feito o acompanhamento de operações de corte de árvores em povoamentos de Eucalyptus grandis com 7 anos de idade, plantados nas regiões de Belo Oriente e Guanhães, MG, pertencentes à Cenibra - Celulose Nipo-Brasileira S.A. (Tabela 1). O diferencial das duas regiões foi quanto à produção da floresta e ao índice de sobrevivência, possibilitando o estudo em regiões com padrões florestais distintos (Tabela 2).

Tabela 1. Descrição das áreas.

Table 1. Area description.

\begin{tabular}{lcc}
\hline Características & Belo Oriente & Guanhães \\
\hline Relevo & $\begin{array}{c}\text { Plano a fortemente ondulado } \\
\text { Solo }\end{array}$ & $\begin{array}{c}\text { Suave a fortemente ondulado } \\
\text { Precipitação média anual }\end{array}$ \\
Temperatura média anual & baixadas férteis & Profundos, argilosose pouco férteis \\
UR média anual & $1.163 \mathrm{~mm}$ & $1.154 \mathrm{~mm}$ \\
Déficit hídrico & $25,2^{\circ} \mathrm{C}$ & $22,2^{\circ} \mathrm{C}$ \\
Altitude média & $65 \%$ & $58 \%$ \\
\hline
\end{tabular}

Tabela 2. Descrição dos povoamentos de Eucalyptus grandis.

Table 2. Eucalyptus grandis plantation characteristics.

\begin{tabular}{lcc}
\hline Características & Belo Oriente & Guanhães \\
\hline Espaçamento $(\mathrm{m})$ & $3 \times 2$ & $3 \times 2$ \\
Sobrevivência & $65 \%$ & $90 \%$ \\
Número de árvores $/ \mathrm{m}^{3}$ & 6,5 & 6,7 \\
Produção média estimada & $165 \mathrm{~m}^{3} \cdot \mathrm{cc}^{2} \cdot \mathrm{ha}^{-1}$ & $224 \mathrm{~m}^{3} \cdot \mathrm{cc}^{-1} \mathrm{ha}^{-1}$ \\
\hline
\end{tabular}

Equipamentos

Utilizaram-se dois modelos de feller-buncher: um Timberjack 608 L (Figura 1), com 15.000 horas de uso, em Belo Oriente, e um Timbco 520 (Figura 2), com 5.000 horas, em Guanhães.

Foram utilizados também um clinômetro, para determinação da inclinação do terreno, uma trena equipada com infravermelho e um binóculo a laser, para determinação da distância do eito de corte, um cronômetro digital marca Cássio modelo HS-30 exatidão 1/100s, prancheta e formulários para coleta de dados de desempenho e estudo de tempos.

\section{Método}

Corte

A operação de corte com feller-buncher foi feita em eitos de quatro linhas de árvores, sendo avaliadas 10 parcelas por classe de inclinação do terreno (Tabela 3), com 100 árvores cada uma. A partir 
de um ponto fixo, o feller-buncher conseguia cortar 4 árvores em 4 linhas em sequência e depois colocálas em uma pilha disposta na diagonal com relação ao sentido de movimentação do skidder. A inclinação do terreno em graus, no local de cada parcela, foi estimada com a utilização do clinômetro, com medidas obtidas a cada $10 \mathrm{~m}$ em uma mesma linha de árvores.

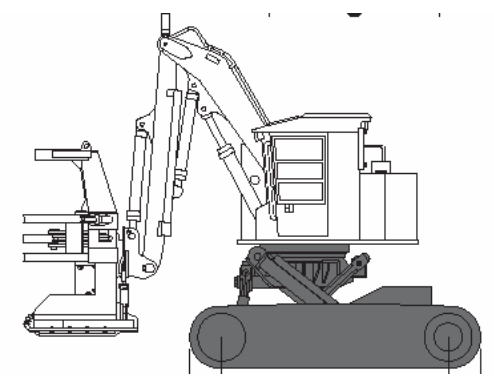

Potência

Alcance da grua

Peso

Nivelamento de cabine

Pressão no solo
$171 \mathrm{~kW}$

$7,10 \mathrm{~m}$

$27 \mathrm{t}$

$27^{\circ}$

$56 \mathrm{kPa}$

Figura 1. Desenho e características técnicas do feller-buncher Timberjack 608L.

Figure 1. Feller-buncher Timberjack 608L technical specifications.

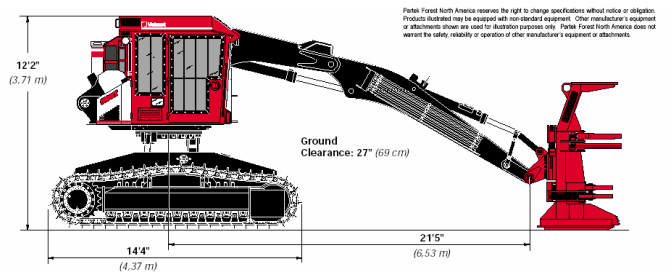

Potência

Alcance da grua

Peso

Nivelamento de cabine

Pressão no solo
$180 \mathrm{~kW}$

$7,10 \mathrm{~m}$

$27 \mathrm{t}$

$27^{\circ}$

$58 \mathrm{kPa}$

Figura 2. Desenho e características técnicas do feller-buncher Timbco 520.

Figure 2. Feller-buncher Timbco 520 technical specifications.

Tabela 3. Classes de inclinação do terreno.

Table 3. Ground level classes.

\begin{tabular}{lcc}
\hline Classe & \% & Grau \\
\hline 1 & $0-10$ & $0-6$ \\
2 & $11-20$ & $7-11$ \\
3 & $21-33$ & $12-18$ \\
4 & $34-50$ & $19-27$ \\
\hline
\end{tabular}

A produtividade da máquina, por hora efetiva de trabalho, foi obtida através de estudo de tempo com o método de tempo contínuo. Nesse estudo, a contagem de tempo foi iniciada no momento do corte da primeira árvore da amostra e finalizada ao final da deposição no solo da centésima árvore cortada e colocada no feixe. Nessa etapa, junto com a análise da dinâmica operacional, foram anotados o número de árvores acumuladas por ciclo de corte e a produtividade do talhão $\left(\mathrm{m}^{3} \cdot \mathrm{ha}^{-1}\right)$, sendo avaliadas apenas as máquinas sem a ocorrência de precipitação (caso houvesse, era interrompida a seção dos testes e iniciada outra nas mesmas condições das anteriores). A análise da influência da produção da floresta sobre o desempenho do feller foi feita também em três talhões com volumes por hectare bem diferenciados.

Em todas as medições, foram escolhidos os operadores de mesmo nível técnico e com experiência média de 6 anos de empresa, desconsiderando-se os novatos e também os mais experientes. Todas as tomadas de tempo foram realizadas no turno das $7 \mathrm{~h} 00 \mathrm{~min}$. às $16 \mathrm{~h} 00 \mathrm{~min}$.

Uma coleta preliminar de 10 amostras por classe de declive e classe de produtividade foi avaliada quanto ao número ideal de amostras a serem coletadas, de acordo com a fórmula:

$$
N^{\prime}=\frac{t^{2} \cdot s^{2}}{(0,1 \cdot \bar{x})^{2}}
$$

(COCHRAN, 1965) 
Em que: $\mathrm{N}^{\prime}=$ número necessário de observações para prever o tempo correto dentro de $\pm 5 \%$ de erro e

95\% de nível de confiança;

$\mathrm{t}=$ valor de tabela ( $\mathrm{t}-$ Student);

$\mathrm{s}=$ desvio padrão;

$\overline{\mathbf{X}}=$ produtividade média do "feller-buncher" $\left(\mathrm{m}^{3} \cdot \mathrm{h}^{-1}\right)$.

De acordo com as amostras preliminares, nove parcelas seriam suficientes para a confiabilidade de $95 \%$, sendo adotadas como padrão 10 amostras por classe. Com posse desses resultados, foi calculada a produtividade por hora efetiva de trabalho em função da inclinação do terreno e determinada uma equação estimando essa relação.

Foi feita a análise da dinâmica operacional através de estudo de tempo com método multimomento (com observações de 15 em 15 segundos), dos seguintes elementos do ciclo operacional observados para o conjunto colhedora e seus movimentos do cabeçote de corte:

- Tempo de deslocamento sem carga.

- Tempo de corte efetivo.

- Tempo de deslocamento entre árvores.

- Tempo de deslocamento para o descarregamento (bascular).

O delineamento estatístico utilizado foi de um experimento inteiramente casualizado, sendo feitas análises em separado para cada uma das duas regiões, considerando-se quatro tratamentos quanto às classes de inclinação do terreno (Tabela 3), com dez repetições por tratamento. A comparação de médias foi feita pelo Teste de Tukey, ao nível de probabilidade de $5 \%(\mathrm{p}<0,05)$. Ao mesmo tempo, efetuou-se uma análise para comparação entre as médias de cada tratamento obtidas nas duas regiões, buscando-se avaliar a influência da produtividade de cada povoamento sobre o desempenho das máquinas.

Buscou-se também a predição geral da produtividade do feller-buncher $\left(\mathrm{m}^{3} \mathrm{~h}^{-1}\right)$ para as duas regiões em função da declividade do terreno (graus) e produtividade da floresta, realizando-se análises de regressão para a determinação de equações que melhor estimassem essa relação. Utilizou-se o programa BioEstat 3.0 para ajustamento de curvas nos modelos de regressão linear, exponencial, logarítmica e geométrica.

\section{RESULTADOS E DISCUSSÃO}

Os resultados expressam o desempenho das máquinas em termos de capacidade de campo efetiva $\left(\mathrm{C}_{\mathrm{C}} \mathrm{E}\right)$ do trabalho dentro dos procedimentos normais adotados na empresa, cuja unidade é expressa em metros cúbicos por hora $\left(\mathrm{m}^{3} \cdot \mathrm{h}^{-1}\right)$. Assim, não foram computadas as horas paradas para manutenção, abastecimento, reparos e pausas pessoais, e sim o tempo realmente gasto na função estabelecida. O registro da precipitação nas duas regiões durante o período do estudo está detalhado na tabela 4, que mostra a similaridade climática de ambas quanto à distribuição pluviométrica. As tomadas de tempo consideradas neste estudo só contemplaram as atividades realizadas sem chuva durante as observações da dinâmica operacional.

Tabela 4. Dados de precipitação mensal por região.

Table 4. Monthly pluviometer data by region.

\begin{tabular}{lcc}
\hline \multirow{2}{*}{ Mês } & \multicolumn{2}{c}{ Precipitação na região (mm) } \\
\cline { 2 - 3 } & Belo Oriente & Guanhães \\
\hline Dezembro & 148,59 & 293,63 \\
Janeiro & 282,70 & 186,43 \\
Fevereiro & 257,05 & 222,51 \\
Março & 152,62 & 119,63 \\
Abril & 146,05 & 137,68 \\
\hline
\end{tabular}

\section{Produtividade do feller-buncher em função das classes de relevo}

A produtividade do feller-buncher apresentou diferenças significativas nas quatro classes de inclinação para as duas regiões e também entre regiões. 
Em florestas de maior produtividade e melhor padrão, observou-se o maior desempenho na operação de corte, verificando-se também as maiores influências do relevo sobre a capacidade de desempenho da colhedora e maiores coeficientes de variação (Tabela 5).

Tabela 5. Média e coeficiente de variação (CV) da produtividade do feller-buncher $\left(\mathrm{m}^{3} \mathrm{~h}^{-1}\right)$ por classe de inclinação e região.

Table 5. Average and coefficient of variation (CV) of feller-buncher productivity $\left(\mathrm{m}^{3} \mathrm{~h}^{-1}\right)$ by inclination class and region.

\begin{tabular}{lcc}
\hline Classe de inclinação & Belo Oriente & Guanhães \\
\hline Classe 1 & 65,9 aa* $(7,6 \%)$ & $95,5 \mathrm{ab}(9,2 \%)$ \\
Classe 2 & $65,5 \mathrm{aa}(5,7 \%)$ & $71,8 \mathrm{bb}(11,0 \%)$ \\
Classe 3 & $41,6 \mathrm{ba}(8,3 \%)$ & $65,7 \mathrm{bb}(7,8 \%)$ \\
Classe 4 & $35,5 \mathrm{ca}(8,9 \%)$ & $47,5 \mathrm{cb}(14,4 \%)$ \\
\hline * A primeira letra indica relação entre classes na mesma região, e a segunda letra entre \\
regiões na mesma classe, sendo que mesma letra indica semelhança estatística ao nível de 5\% \\
de probabilidade pelo teste de Tukey.
\end{tabular}

Nota-se que a produtividade da máquina em florestas de baixo padrão (Belo Oriente) teve menor variação se comparada à de melhor padrão (Guanhães), indicando serem mais pronunciadas as perdas de desempenho quanto maior for o padrão da floresta.

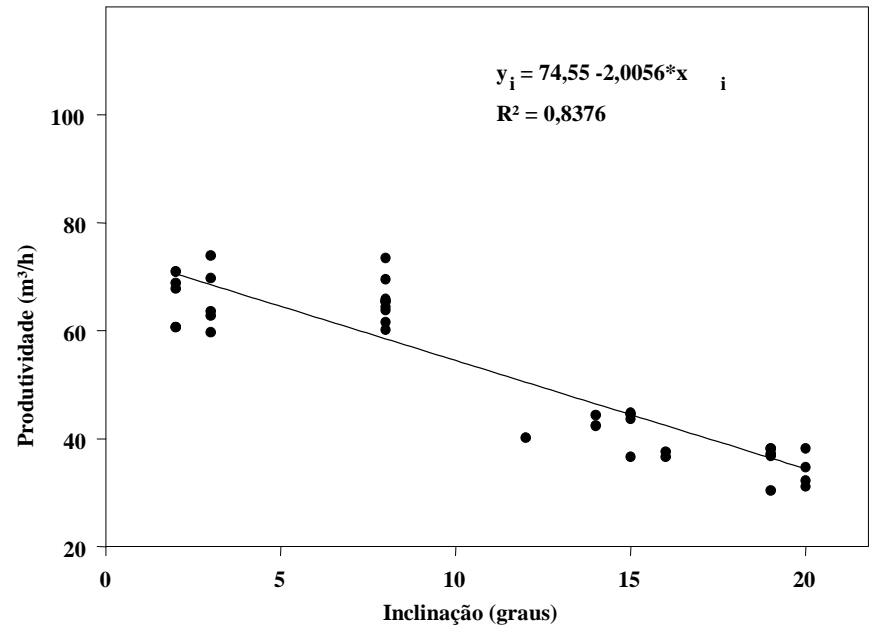

Figura 3. Regressão linear para produtividade do feller-buncher em função da inclinação na região de Belo Oriente.

Figure 3. Linear regression of feller-buncher productivity as a function of inclination at Belo Oriente.

A análise de variância feita isoladamente para a região de Belo Oriente detectou diferença significativa entre as classes de inclinação do terreno, com exceção entre a $1^{a}$ e a $2^{a}$. Escolheu-se o modelo de regressão linear, que explicou $84 \%$ da variabilidade da variável resposta (Figura 3), sendo a produtividade inversamente proporcional à inclinação. Contudo, a distribuição dos dados coletados para a Classe 2 (7-11 graus) talvez levasse a um outro modelo, desde que utilizada uma análise de regressão mais complexa, o que não foi considerado essencial para este estudo.

Na região de Guanhães também houve diferenças significativas entre as classes de inclinação, excetuando-se a semelhança entre as classes 2 e 3 . Nesse caso, o modelo mais adequado foi o de regressão logarítmica, explicando $78 \%$ da variabilidade da variável resposta (Figura 4). Nessa região, a queda de produtividade foi mais acentuada, o que talvez pudesse ser explicado pela maior produtividade da floresta, em função da dificuldade de manuseio de árvores maiores em terreno mais acidentado.

$\mathrm{O}$ estudo de tempo forneceu informação extra sobre a dinâmica operacional do feller-buncher, sendo destacados na tabela 6 os resultados dos dois extremos em termos de inclinação do terreno: classe 1 e classe 4 .

Nota-se que na classe 1 houve maior tempo gasto para buscar as árvores (deslocamento entre árvores) na região de Belo Oriente e menor para a região de Guanhães, indicando haver influência da 
porcentagem de falhas do plantio sobre o tempo. No entanto, quanto mais acidentado o relevo menor foram essas diferenças. $\mathrm{O}$ tempo de deslocamento do feller-buncher com árvores cortadas acumuladas no cabeçote é maior na região mais acidentada, não tendo sido definido o motivo desse fato.

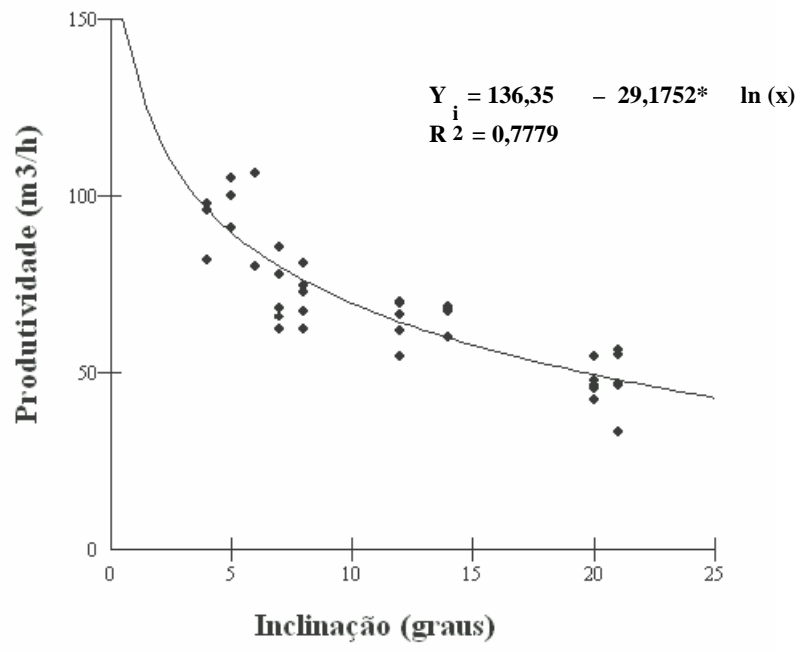

Figura 4. Regressão logarítmica para produtividade do feller-buncher em função da inclinação do terreno na região de Guanhães.

Figure 4. Logarithmic regression of feller-buncher productivity as function of inclination at Guanhães.

Tabela 6. Distribuição percentual de tempos e movimentos do feller-buncher nos extremos de inclinação do terreno nas regiões de Belo Oriente e Guanhães.

Table 6. Feller-buncher work and time study at two extreme ground inclination classes at Belo Oriente and Guanhães regions.

\begin{tabular}{lcccc}
\hline \multirow{2}{*}{ Atividade } & \multicolumn{2}{c}{ Classe 1 } & \multicolumn{2}{c}{ Classe 4 } \\
\cline { 2 - 5 } & Belo Oriente & Guanhães & Belo Oriente & Guanhães \\
& $\mathbf{\%}$ & 21 & $\boldsymbol{\%}$ & 23 \\
\hline Deslocamento sem carga & 14 & 35 & 22 & 27 \\
Corte & 27 & 29 & 34 & 33 \\
Deslocamento entre árvores & 44 & 15 & 16 & 17 \\
Bascular & 15 & & & $\%$ \\
\hline
\end{tabular}

Complementando-se o estudo, foi feito o comparativo do desempenho do feller-buncher em áreas planas, variando-se apenas o padrão de floresta (Tabela 7). A floresta considerada de melhor padrão nas regiões estudadas foi a do projeto Marcocem, com produção de $264 \mathrm{~m}^{3}$.ha ${ }^{-1}$ e $90 \%$ de sobrevivência, seguida do projeto Lagoa Grande I, com $200 \mathrm{~m}^{3} \cdot \mathrm{ha}^{-1}$ e $75 \%$ de sobrevivência e, finalmente, o projeto Tamanduá, com cerca de $164 \mathrm{~m}^{3} \cdot \mathrm{ha}^{-1}$ e $60 \%$ de sobrevivência. O modelo de regressão linear obtido explicou $90 \%$ da variabilidade da produtividade da máquina $\left(\mathrm{m}^{3} \cdot \mathrm{h}^{-1}\right)$ em função do padrão da floresta $\left(\mathrm{m}^{3} \cdot \mathrm{ha}^{-1}\right)$ (Figura 5).

Como constatado na tabela 7, o volume individual por árvore influi positivamente na capacidade operacional, concordando com os trabalhos de demais autores (GINGRAS, 1988; VALVERDE et al., 1996; VISSER; STAMPFER, 2003).

Pode-se pressupor que o tempo gasto para cortar uma árvore de $0,11 \mathrm{~m}^{3}$ seja igual ao de outra de $0,25 \mathrm{~m}^{3}$, no entanto, ao se considerar a capacidade produtiva em termos volumétricos, somada à inclinação do relevo e produção da floresta, essa relação é de difícil predição. O modelo deste estudo possibilitou predizer o desempenho médio da colhedora denominada feller-buncher tendo-se como entradas a inclinação do relevo e a produção da floresta, aumentando as possibilidades do planejamento de tempo de permanência da equipe de colheita em determinado padrão de floresta de acordo com o relevo predominante. 
Tabela 7. Resumo estatístico para as variáveis estudadas de desempenho do feller-buncher na operação de corte em área plana.

Table 7. Statistical results of feller-buncher productivity variables on flat terrain.

\begin{tabular}{lccccc}
\hline Projeto & Estatística & ${\text { Arv. } \mathbf{m i n}^{-\mathbf{1}}}^{-}$ & $\mathbf{A r v . m}^{-\mathbf{3}}$ & $\mathbf{~ m}^{\mathbf{3}} \cdot \mathbf{h}^{-\mathbf{1}}$ & Arv./ciclo \\
\hline Marcocem & Média (S) & $8,77( \pm 0,55)$ & 4,5 & $116,19( \pm 7,35)$ & $6,8( \pm 0,4)$ \\
(Belo Oriente) & $\mathrm{CV}$ & $6,2 \%$ & - & $6,3 \%$ & $5,8 \%$ \\
Lagoa Grande I & Média (S) & $7,54( \pm 0,80)$ & 6,7 & $67,57( \pm 7,22)$ & $5,8( \pm 0,7)$ \\
(Guanhães) & $\mathrm{CV}$ & $10,6 \%$ & -- & $10,7 \%$ & $12 \%$ \\
Tamanduá & Média (S) & $6,64( \pm 1,04)$ & 7,2 & $55,35( \pm 8,62)$ & $6,4( \pm 0,9)$ \\
(Belo Oriente) & $\mathrm{CV}$ & $15,6 \%$ & -- & $15,6 \%$ & $14 \%$ \\
\hline
\end{tabular}

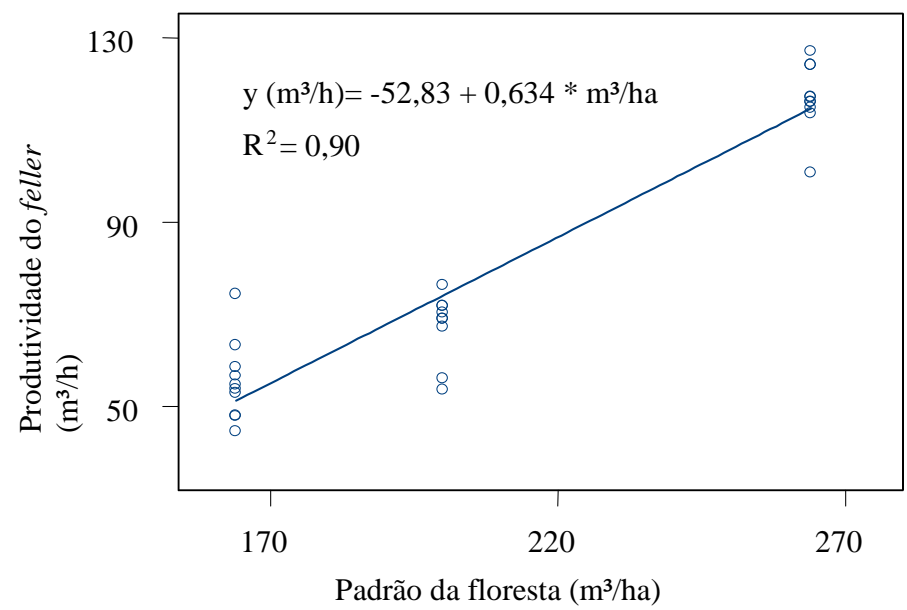

Figura 5. Modelo de regressão linear para a produtividade da máquina feller-buncher em função da produção da floresta.

Figure 5. Linear regression of feller-buncher productivity as a function of forestry productivity.

A análise conjunta da influência das variáveis inclinação do terreno e produtividade da floresta sobre a produtividade do feller-buncher, considerando-se todos os dados obtidos neste estudo nas regiões de Guanhães e Belo Oriente, resultou na equação 1.

$$
P_{F B}=37,04-1,52 \cdot I+0,23 \cdot P f \quad\left(\mathrm{R}^{2}=0,72\right)
$$

Em que: $\mathrm{P}_{\mathrm{FB}}=$ produtividade do feller-buncher $\left(\mathrm{m}^{3} \cdot \mathrm{h}^{-1}\right)$;

$\mathrm{I}=$ inclinação do terreno $\left(^{\circ}\right)$;

$\mathrm{PF}=$ produtividade da floresta $\left(\mathrm{m}^{3} \cdot \mathrm{ha}^{-1}\right)$.

Outros fatores também devem afetar a produtividade do feller-buncher, como o operador, a idade da máquina e o sistema de corte, sendo que, nessas condições, os principais elementos foram a inclinação do terreno e a produtividade da floresta, explicando-se, pela modelagem feita, $80 \%$ e $90 \%$ da variação, respectivamente. De modo geral, $72 \%$ das variações foram dadas pelos dois principais fatores contemplados no modelo.

A operação corte de árvores com feller-buncher demonstrou ser fortemente influenciada pela inclinação do terreno, com o aumento da inclinação afetando entre $67 \%$ e $82 \%$ da resposta quanto à produtividade da máquina, de acordo com a produção menor ou maior da floresta, respectivamente.

\section{CONCLUSÕES}


- A operação de corte de árvores com feller-buncher foi significativamente influenciada pela inclinação do terreno em qualquer padrão de floresta. A queda da produtividade do feller-buncher, conforme a classe de inclinação, foi inversa, perdendo nas áreas de maior inclinação do terreno cerca de metade da produtividade daquela obtida em terreno plano, para ambas as regiões estudadas. $\mathrm{Na}$ análise isolada da influência do padrão da floresta, mantendo-se o corte somente em áreas em terreno plano, a produtividade do feller-buncher foi diretamente proporcional à melhoria do padrão da floresta em termos de volume por hectare.

- Com a predição da produtividade da colhedora, é possível determinar o tempo real gasto para a colheita em cada projeto, tendo-se como pré-requisitos o padrão de floresta e a inclinação média de cada talhão. Algo simples de ser calculado a partir dos dados amostrados e analisados antes da chegada da equipe de corte.

\section{AGRADECIMENTOS}

Os autores agradecem à Cia. Cenibra (Celulose Nipo-Brasileira S.A.), na pessoa do Engenheiro Maurício Muramoto, pelo total apoio para a realização deste trabalho.

\section{REFERÊNCIAS}

COCHRAN, W. G. Técnicas de amostragem. Rio de Janeiro: Fundo de Cultura, 1965. 555 p.

CONWAY, S. Logging practices: principles of timber harvesting systems. San Francisco: Miller Freeman, 1976. 416 p.

GINGRAS, J. F. The effects of site and stand factors on feller-buncher performance. Feric Technical Report, Vancouver, n. 84, p. 1-18, nov. 1988.

MALINOVSKI, J. R.; CAMARGO, C. M. S.; MALINOVSKI, R. A. Sistemas. In: MACHADO, C.C. (Ed.) Colheita florestal. Viçosa, MG: UFV, 2002. p. 145-67.

SANT'ANNA, C. M. Corte florestal. In: MACHADO, C.C. (Ed.) Colheita florestal. Viçosa, MG: UFV, 2002. p. 55-88.

VALVERDE, S. R.; MACHADO, C. C.; REZENDE, J. L. P.; SOUZA, A. P.; ANTIQUEIRA, A. C. Análise técnico-econômica do corte de madeira com o trator florestal derrubador-amontoador (fellerbuncher) no sistema de colheita florestal de árvores inteiras de eucalipto. Revista Árvore, Viçosa, MG, v. 20, n. 2, p. 229-40, 1996.

VISSER, R.; STAMPFER, K. Tree-length system evaluation of second thinning in a loblolly pine plantation. Southern Journal of Applied Forestry, Bethesda, v. 27, p. 77-82, 2003. 